\title{
An integrated approach to modelling fish cage response in the flow
}

\author{
S. Gao ${ }^{1}$ L. C. Gansel ${ }^{1}$ G. Li $^{2}$ H. Zhang ${ }^{2}$ \\ ${ }^{1}$ Department of Biological Sciences, Norwegian University of Science and Technology, N-6025 Ålesund, Norway. \\ E-mail: Sihan.Gao@ntnu.no \\ ${ }^{2}$ Department of Ocean Operations and Civil Engineering, Norwegian University of Science and Technology, N-6025 \\ Alesund, Norway.
}

\begin{abstract}
Noticeable progress has been made in recent years regarding sensor-based monitoring and model-based simulation of sea cage response. The discrepancy between measured data and simulation results can cause confusion about the actual deformation of fish cages. This study aims to explore an approach of integrating measured depth data with a deterministic cage model for sea cage response prediction in dynamic analysis software Orcaflex, emphasizing on cage deformation estimation. A Morison-type cage model is divided into several net panels, regulators based on PID theory are developed and incorporated into the cage model to regulate the hydrodynamic properties of cage net panels based on measured depth. Through a case study based on published model tests, it is shown that the model incorporating measured depth can significantly improve the predictions of cage deformation compared with deterministic models. The resultant drag forces from the integrated models are also with reasonably good accuracy.
\end{abstract}

Keywords: fish cage modelling, Morison-type load model, fish cage deformation, fish cage digitalization

\section{Introduction}

Net cages are commonly used as production units in farming of salmonids. A net cage is usually constrained by floaters at the top and a weight system connecting to the bottom to keep the cage shape and position stable. In many farming regions, currents can change hugely at different time scales, often from tidal cycles to seasons, leading to a significant variation of hydrodynamic loads over time. This substantial variability may cause significant deformation of fish cages. Field experiments on a farm site in Norway show an estimated volume reduction of $30 \%$ of a commercial salmon cage subjected to a current of $0.6 \mathrm{~m} / \mathrm{s}$ Klebert et al. (2015).

A good understanding of sea cage deformation is of great importance, as significant volume reduction of fish cages can increase stress levels of the stocked fish and adversely affect their growth and health Turnbull et al. (2005), and regulations usually impose a maximum stocking density. In addition, a good prediction of cage shape is indispensable for the development of modern aquaculture techniques, as it facilitates the use of autonomous underwater vehicles (AUVs) for cage inspection through clarifying relative position $\mathrm{Su}$ et al. (2021). More efficient fish pellet delivery control can be achieved by considering deformed cage shapes. Moreover, cage deformation is highly coupled with ambient flow fields. Fluid movement induces cage deformation, and cage structures disturb the incoming flow Gansel et al. (2012). Therefore observing cage deformation can provide new insights into the flow field in and around fish cages.

Several knowledge-based numerical cage models have been developed and studied to predict fish cage deformation under currents in previous research. A typical full-sized fish cage contains more than one million 
meshes; therefore, direct modelling of a fish cage is computationally inefficient. Equivalent structure models such as the truss model Tsukrov et al. (2003), massspring model Lee et al. (2005), and triangular element model Priour (1999) have been proposed to group net twines into simple structures. Fluid loads acting on the cage net structure can be calculated based on Morison's equation Tsukrov et al. (2003); Moe et al. (2010); Cifuentes et al. (2015) or by screen-type models Kristiansen and Faltinsen (2012); Løland (1993). Shielding effects of the upstream half cage on the incoming current can significantly reduce the fluid loads on the downstream part of the cage, and thus wake models were given to estimate the flow reduction inside fish cages Aarsnes et al. (1990); Cifuentes and Kim (2017).

Experimental methods were also applied to investigate the hydrodynamic behavior of fish cages. There have been lab experiments on scaled cage models, where cage deformation exposed to uniform flow was captured through underwater video systems Lader and Enerhaug (2005). Acoustic transmitters Su et al. (2021); DeCew et al. (2013) or pressure tags Klebert et al. (2015); Gansel et al. (2018); Lader et al. (2008) were applied in field experiments to observe cage deformation in a more realistic scenario. The reduced flow velocity inside cages was measured through Vector Acoustic Doppler Velocimeters (ADVs) and Acoustic Doppler Current Profilers (ADCPs) Klebert et al. (2015); DeCew et al. (2013); Gansel et al. (2018) and the fluid loads on net cages were measured through tension transducers Lader and Enerhaug (2005); Gansel et al. (2018).

Previous research has been extensive, but in practice it is challenging to predict the actual cage shape through either sensory or simulation techniques. Although observation of fish cage deformation based on sensor data can be reliable and real-time updating can be achieved via wireless transmission, acoustic transmitters can only provide position data where they locate, and pressure tags only provide depth information. Compared with the real measurement, numerical simulations can predict cage shapes in detail, but the accuracy of simulation results is strongly dependent on the selection of hydrodynamic parameters and validity ranges of numerical models Gansel et al. (2018); Cheng et al. (2020); Moe-Føre et al. (2015). Besides, fish cages at farm sites experience complex interactions with environmental and biological factors, such as dramatic flow field changes in fish cages at high flow speed Gansel et al. (2012, 2014), different physical properties between wet and dry cage net Moe et al. (2010), and biological influences such as biofouling accumulation Gansel et al. (2017); Fitridge et al. (2012) and the fish inside the cage He et al. (2018). These factors cannot

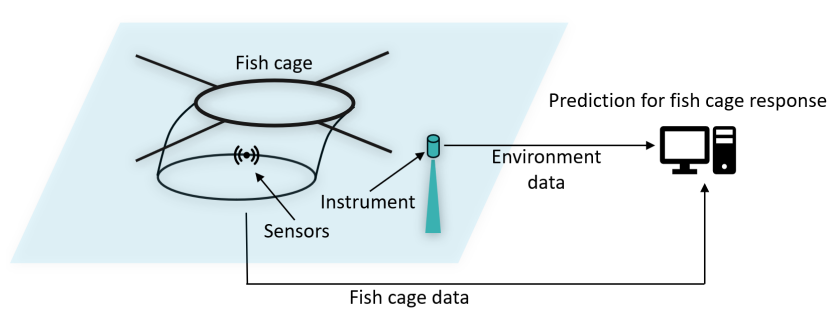

Figure 1: Concept of online cage response monitoring

be completely considered in the simulation. The normal way to handle them is to simplify the model conservatively. Although it is suitable for design purposes, too much simplification in modeling will broaden the gap between simulation and reality.

Combing sensor data with deterministic models should be a way to handle the uncertainties. The concept is shown in Fig. 1. Various sensors will be applied to collect cage response information, and the surrounding sea condition will be measured by instruments such as ADCPs. Onsite signals from sensors and instruments will be transmitted to simulators in real-time, where these measured data will be processed and used to calibrate numerical models for cage response prediction and the output will be visualized for monitoring purpose.

In this study, we investigated the feasibility of integrating sensor data (measured depth) with a Morisontype cage model via Proportional-Integral-Derivative (PID) regulators for net cage deformation prediction. The results show that the integrated models can predict cage deformation closer to measurements than the deterministic models, through regulating the hydrodynamic parameters of the cage net based on sensor data. Key contributions of the paper include the development of an integrated model for cage deformation prediction, test of the ability of PID regulators regarding identifying the hydrodynamic parameters of net cages, and implementation of the integrated model based on a published experiment Lader and Enerhaug (2005).

The remainder of the paper is structured as follows: Section 2 introduces the general scheme of the integrating method and the details of creating cage models and PID regulators. Section 3 presents the implementation of the proposed method based on a published model test Lader and Enerhaug (2005), including feasibility studies and integrating the measured data with the numerical models. Section 4 presents and discusses the experiment results. Section 5 summarizes the findings and offers directions for future research. 


\section{Integrating sensor data with a deterministic model}

\subsection{General scheme}

Changes in currents at a farm site contain low frequency components, largely because of tidal variation, and high frequency components because of waves and vortices. Unlike the high frequency components, low frequency components primarily affect the global net cage deformation. Therefore, cage deformations in a current can be resolved in a quasi-dynamic way, where the current velocity and corresponding cage response are updated by an approximation of stability over a particular period of time.

Fig. 2 illustrates the general scheme of the integrating method. Within each time interval, sensor data regarding depth information and current velocity is collected and processed first. The initial simulation is based on a deterministic model using default hydrodynamic parameters from empirical formulas, and the measured current velocity is input as the updated environmental conditions. The initial simulation results will be compared with the measured depth of the net cage, and the discrepancy between measurements and simulation will be obtained. When the discrepancy is out of an acceptable error margin, the discrepancy will be input into the PID regulators, which adjust the hydrodynamic parameters of net panels to minimize the discrepancy. The adjusted hydrodynamic parameters will be input into the next round of simulations, and this will repeat until an acceptable agreement between simulation and measurements at all sensor points. The numerical model with the final tuned-up hydrodynamic parameters will be utilized to estimate the response of the cage under the current.

\subsection{Deterministic cage mode}

\subsubsection{Structure and fluid load model}

Truss elements were used to simplify the net structures Tsukrov et al. (2000). Fig. 3a shows a net panel represented by several truss elements. Each truss element was assigned with a projected area, submerge weight, and elasticity equal to the sum of parallel twines within the width of an equivalent mesh Tsukrov et al. (2000) so that the hydrodynamic performance of the net made up of trusses can be similar to the original one. To better reflect the nature of flexible net twines, the truss elements were split into several segments to allow for buckling. A neglectable stiffness was added to the segment joints to improve the truss elements' determinacy under buckling.

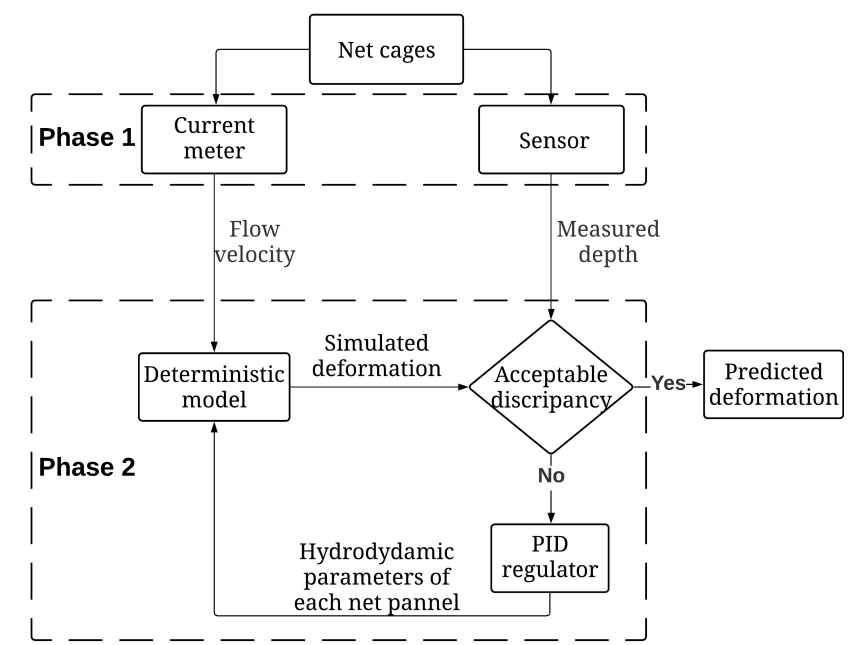

Figure 2: The general scheme of integrating measured depth data with a deterministic cage model.

Since the cage response was simulated in a quasidynamic way, the fluid load acting on each truss element was calculated using the drag term of Morison's equation. The incident flow $V$ was decomposed in directions parallel $V_{T}$ and normal $V_{N}$ to the element following the cross-flow principle (Fig. 3b). Compared with the normal force component $F_{N}$, the frictional force in the tangential direction is minor Cheng et al. (2020); Moe-Føre et al. (2016), and thus was neglected. The magnitude of $F_{N}$ was calculated by Eq. 1:

$$
F_{N}=\frac{1}{2} C_{D} \rho D L V_{N}^{2}
$$

where $C_{D}$ is the drag coefficient, $\rho$ is the liquid density, and $D$ and $L$ are the diameter and length of the truss element. The normal force component $F_{N}$ can be further decomposed into two directions: the force component in the flow direction is drag force $F_{D}$, and the one normal to the flow is lift force $F_{L}$.

The downstream part of a net cage is subject to a reduced flow velocity due to the shielding effects of the upstream net. In this study, numerical cage models were subjected to a uniform flow field $V$, while the effects of reduced flow on the downstream cage was taken into account by introducing equivalent drag coefficients $C_{D E}$.

$$
C_{D E}=r^{2} C_{D}
$$

where $r$ is the flow reduction factor, which is equal to the ratio of reduced flow velocity inside the cage to incident flow velocity $V$. 


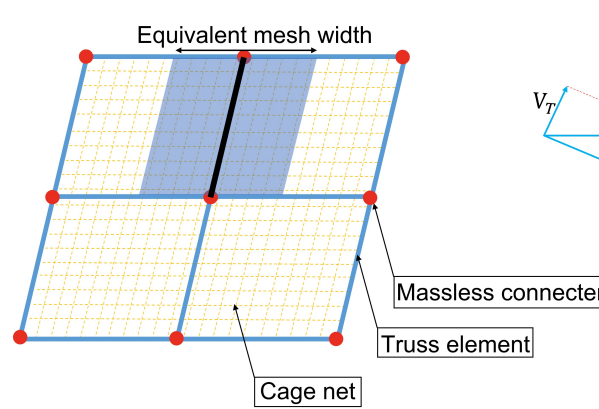

( a )

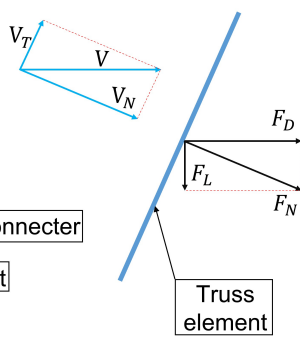

(b)
Figure 3: The numerical model of netting: (a) The structural representation of a net panel as a group of equivalent truss elements. The truss element in black represents the actual net twines in the vertical direction within an equivalent mesh width; (b) The fluid load acting on a truss element.

\subsubsection{Net division}

The imperfection of the Morison-type load model and wake model inside the net cage can widen the deviation between model-based predictions and the measured data, as discussed in Kristiansen and Faltinsen (2012); Moe et al. (2010); Cheng et al. (2020). In the Morison-type load model, the drag coefficient $C_{D}$ is defined as a constant or a function dependent on Reynolds number $R_{e}$ and net solidity $S_{n}$ by different formulas Tsukrov et al. (2003); Cifuentes et al. (2015), which indicates a consistent $C_{D}$ is assigned over the cage. However, according to model tests on net panels, drag loads on the net depend on the attack angles of the flow, especially when the angles are less than $20^{\circ}$ Cheng et al. (2020). This dependency can be explained by the local wake between twines.

In the present method, the net cage was divided into several net panels with their $C_{D}$ defined by regulators, reflecting the fact that fluid loads on the net vary in different parts of the cage. Fig. 4 shows two configurations of net division being implemented. Configuration 1 split the net pen into two parts, the upstream and downstream parts, to consider the upstream's shielding effects. In Configuration 2, the cage was divided into upstream, downstream, and two side parts. This took account of the velocity reduction inside the cage and the influence of the low attack angle.

\subsection{Hydrodynamic parameter regulator}

When a net cage model is created in engineering software, its exact mathematical model is usually sealed and seen as a black box. Thus, traditional gradientbased optimization is not suitable for this case. Regu-
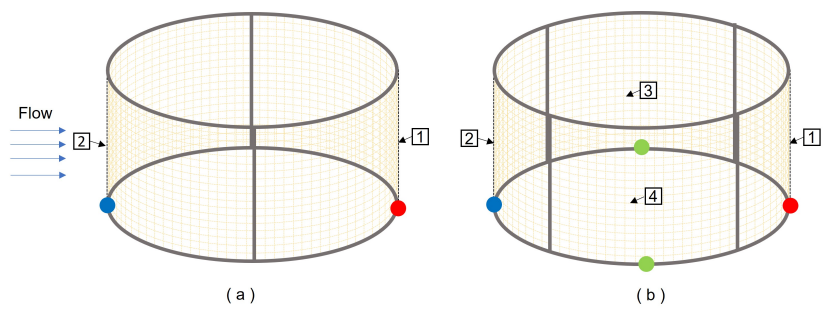

Figure 4: Two configurations of splitting up a circular net pen and the corresponding arrangement of positioning sensors. (a) A net cage equally divided by two as an upstream part (panel 2) and a downstream part (panel 1); (b) A net cage equally divided by four as an upstream part (panel 2), a downstream part (panel 1) and two side parts (panel 3 and 4)

lators based on PID theory can be developed to minimize the simulation results and measurements without knowing the exact mathematical model since there is a correlation between the hydrodynamic parameters of net panels and the simulated net deformation.

As illustrated in Fig. 5, a net panel with a constraint at the top is exposed to the incident flow, which causes the net to deform. If there is a discrepancy between the measured and simulated deformation, it is quantified as a difference in the positions of a spot on the net bottom as $E_{\text {total }}$. $E_{\text {total }}$, decomposed into a horizontal component $E_{x}$ and a vertical component $E_{z} . E_{z}$, representing the difference in the depth direction, was used as the error in the PID regulator. An increasing drag force causes larger deformation and less depth at the bottom point, and vice versa. Thus, a regulating loop can be created to minimize the difference between measured and simulated depth by adjusting the net panel's drag coefficient. In the 2-panel configuration, two separated PID regulators tuned the parameters of the upstream and downstream parts based on the measured depth at the middle bottom of the corresponding net panels (Fig. 4 (a)). In the 4-panel configuration, the two side parts shared the same regulator due to symmetry (Fig. 4 (b)).

\section{Experiment}

The proposed approach was implemented based on a model test presented in Lader and Enerhaug (2005) with the intention of testing the models in a more controlled environment than actual farm sites. Besides, both vertical and horizontal position information was collected from the model test. Part of the measured depths were integrated with numerical models, and the 


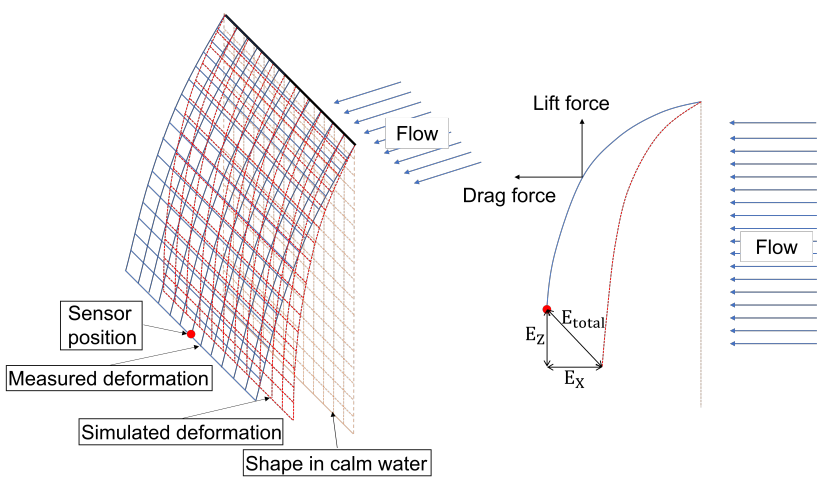

Figure 5: Deformation of net panel under current loads. $E_{\text {total }}$ represents the total positional discrepancy on the middle bottom point of the panel. $E_{Z}$ and $E_{X}$ are the components of $E_{\text {total }}$ in vertical and horizontal directions respectively.

remaining depth data and the horizontal positions were used for evaluation purposes.

In the model test, the cage model was fully immersed in a flume tank and exposed to uniform current at different speeds. Nine markers were placed evenly on one side of the cage model, as shown in Fig. 6, and a video system measured their positions. The processed marker positions presented in Moe et al. (2010) were used in this paper.

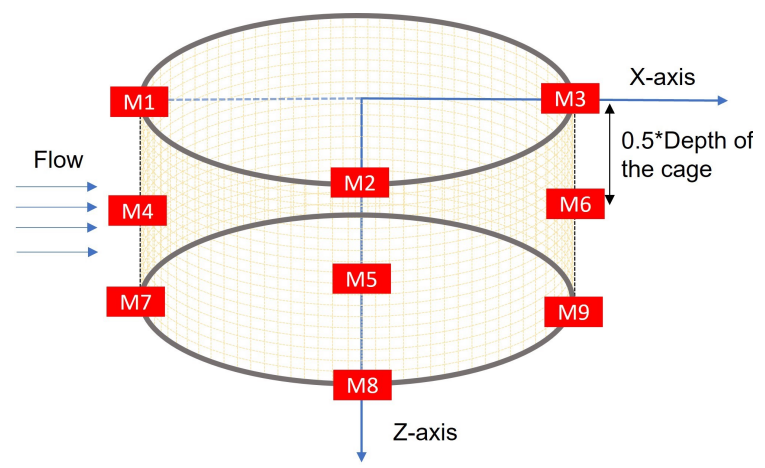

Figure 6: Coordinate system of the net cage model; the M1 to M9 in the red squares are the locations of the nine markers on the cage.

Several cases with different weight configurations were tested in the experiment Lader and Enerhaug (2005). As the influence of weight arrangements was not the focus of this study, and the lightest ballast could lead to the largest cage deformation and reduce the relative measurement error, only the cases of lightest weight configuration $(16 \times 400 \mathrm{~g})$ were used. A summary of the lab test setup is shown in Table 1, and detailed information can be found in Moe et al. (2010);
Table 1: Summary of model test setup Lader and Enerhaug (2005).

\begin{tabular}{lr}
\hline \hline \multicolumn{2}{c}{ Cage structure } \\
\hline Diameter [m] & 1.435 \\
Depth [m] & 1.41 \\
Ballast configuration & $16 \times 400 \mathrm{~g}$ \\
\hline \multicolumn{2}{c}{ Netting configurations } \\
\hline Netting type & Knotless Rasche \\
Material density [kg/m3] & 1130 \\
Nominal bar length [mm] & 17.6 \\
Twine thickness $[\mathrm{mm}]$ & 2 \\
Solidity & 0.225 \\
\hline \hline
\end{tabular}

Lader and Enerhaug (2005).

\subsection{Model creation}

The deterministic cage model in the experiment was built in Orcaflex, a well-developed software for modelling dynamic systems in maritime engineering. The integration of measured data and model-based prediction, including extracting simulation results, net parameter regulating, and results logging, was implemented in a Python environment. Communication between Orcaflex and Python was achieved via an Application Programming Interface (API) Heffernan (2020).

The netting of the cage model was built by the Line model in Orcaflex following the twine grouping approach mentioned earlier. The Line model is represented as a sequence of lamped mass connected by massless springs, suitable for modelling slender structures. 3D buoy models, as point elements with only translational degrees of freedom in three directions, were assigned with a neglectable mass, volume, and exempted from fluid loads. They were allocated to the intersections of the equivalent meshes as joints, and put together the Line models with a common translational motion. The weights of the cage were represented by $3 \mathrm{D}$ buoy models with proper submerge weight and connected to the corresponding mesh joints according to the experiment setup. The drag coefficient for the upstream part of the cage was set as 1.15 , which was recommended by Moe et al. (2010). According to Lader and Enerhaug (2005), the measured flow velocity inside the cage was about $80 \%$ of the incident flow. This shielding effect on the downstream net was introduced in the deterministic model by assigning a reduction factor of 0.64 to the drag coefficient of the downstream half cage following Eq. 2. The drag coefficient of the cage weights was set as 1.1.

The mesh joints at the top were fixed at their original locations to mimic the cage top being constrained 
by a rigid hoop in the experiment. Reaction forces on each of the top joints in $\mathrm{X}$-axis (flow direction) were summed. The drag force on the cage model was calculated as the total reaction force in $\mathrm{X}$-axis minus the drag loads on the weights to keep consistent with the model test results.

Three mesh configurations with increasing mesh numbers from $32 * 10$ to $48^{*} 15$ to $64^{*} 20$ were tested in the simulations with flow speed of $0.33 \mathrm{~m} / \mathrm{s}$ (Fig. 7). The resultant deformation is shown in Fig. 8, and their corresponding drag force and time cost are shown in Table 2. The predicted cage shape from the deterministic model was qualitatively similar to the cage profile captured in the model test. The difference in mesh sizes had a negligible influence on the predicted cage shape and resultant loads, while it affected the computational time significantly. As the present method used iterative ways to resolve cage deformation, an efficient calculation was necessary; thus, the mesh configuration of $32^{*} 10$ was applied in the following studies.

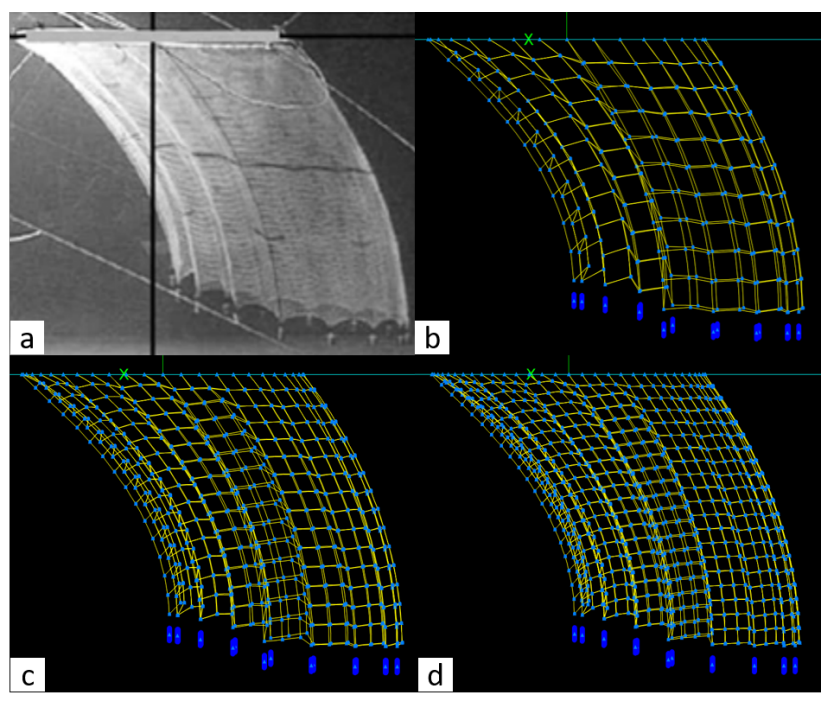

Figure 7: Cage deformation under flow speed of 0.33 $\mathrm{m} / \mathrm{s}$ captured from (a) model tests Lader and Enerhaug (2005), and numerical simulations with different mesh configurations: (b) $32 * 10$, (c) $48^{*} 15$, (d) $64 * 20$.

Table 2: Results from numerical models with different mesh configurations.

\begin{tabular}{ccc}
\hline \hline Mesh number & Drag force $[\mathrm{N}]$ & Time cost [min] \\
\hline $32^{*} 10$ & 43.83 & 0.5 \\
$48^{*} 15$ & 43.86 & 3 \\
$64^{*} 20$ & 43.64 & 9 \\
\hline \hline
\end{tabular}

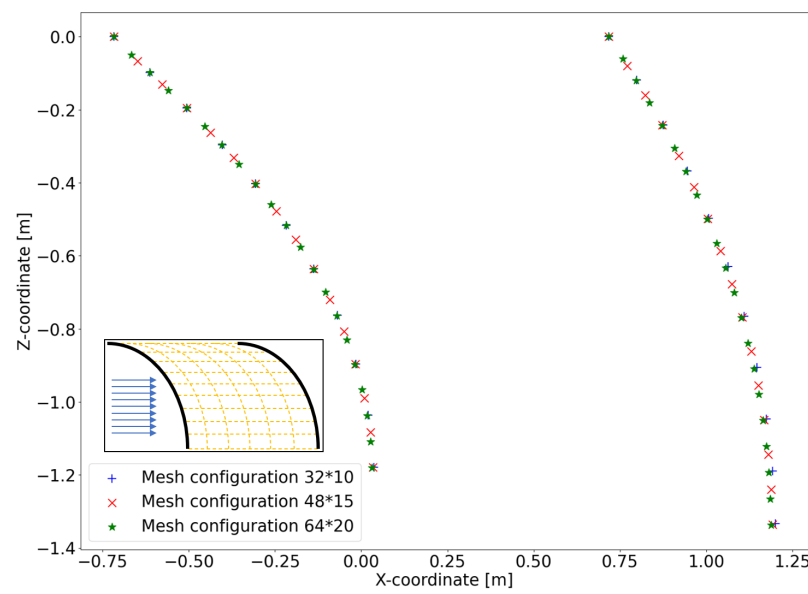

Figure 8: Deformed cage cross-sections from models with different mesh configurations.

Table 3: Convergence study setup.

\begin{tabular}{lr}
\hline \hline \multicolumn{2}{c}{ Environment condition } \\
\hline \multicolumn{2}{c}{ Plow speed [m/s] } \\
\hline \multicolumn{2}{c}{ Prescribed drag coefficient } \\
\hline Upstream panel (Configuration 1, 2) & 1.15 \\
Downstream panel (Configuration 1, 2) & 0.74 \\
Side panels (Configuration 2) & 0.5 \\
\hline \multicolumn{2}{c}{ Initial drag coefficient } \\
\hline Case 1 Case 2 & 0.5 \\
Case 3 & 0.9 \\
\hline \hline
\end{tabular}

\subsection{Identify $C_{D}$ of the net cage through PID regulators}

A convergence study was performed to evaluate the effectiveness of PID regulators to identify cage hydrodynamic parameters from measured data for the two cage division configurations. The setup of the convergence study was shown in Table 3. Dummy measured depths were extracted from simulations of a deterministic model with prescribed hydrodynamic coefficients. Before the regulation, the net cage model was assigned hydrodynamic parameters different from these prescribed values as an initial disturbance. Three cases with some extreme values were performed for the two cage division configurations to test the resolving ability of the PID regulators. A fixed iteration step of 12 was set for one parameter identification process.

Fig. 9 and 10 illustrate the process of drag coefficients of net panels being adjusted to their prescribed values for the 2-panel and 4-panel configuration, respectively. The PID regulators were capable of resolving the exact drag coefficient for each net panel 
within nine steps approximately, despite the different initial settings. The mechanism of PID regulator makes a quicker convergence to the target value when facing larger difference so that the convergence efficiency was not sensitive to the initial values. The regulators should reach convergence faster through customized optimization, but considering the time cost for 12 steps of iterations was typically $6-10$ minutes, a generic setup of PID regulators should be acceptable.

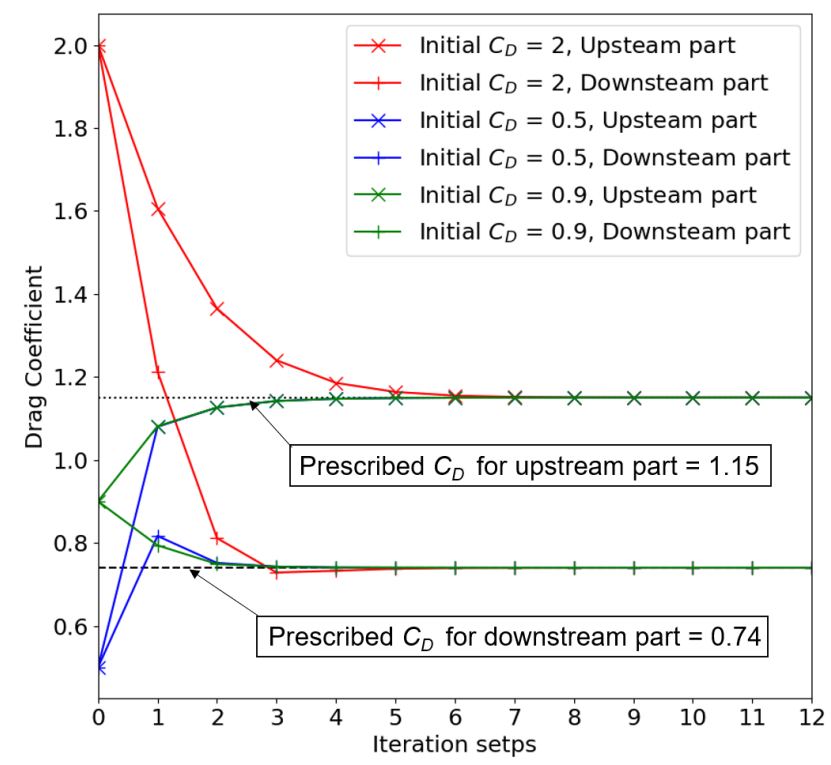

Figure 9: Drag coefficients identified by the PID regulators for the 2-panel configuration.

\subsection{Integrating experiment data with models}

The deterministic model and PID regulators tested in the above section were finally validated using the model test results. Two test cases at moderate $(0.33 \mathrm{~m} / \mathrm{s})$ and high $(0.56 \mathrm{~m} / \mathrm{s})$ flow speed were performed. The criterion of finishing iteration was set as the relative errors (absolute value of error divided by measured depth) at all the fused markers being less than $1 \%$. The measured depth was integrated with the deterministic model, while the measured positions in the horizontal direction were only used for evaluation purposes.

\section{Experiment results}

\subsection{Net cage deformation}

Fig. 11 and 12 compare the cage deformation at moderate speed $(0.33 \mathrm{~m} / \mathrm{s})$ and high speed $(0.56 \mathrm{~m} / \mathrm{s})$. The

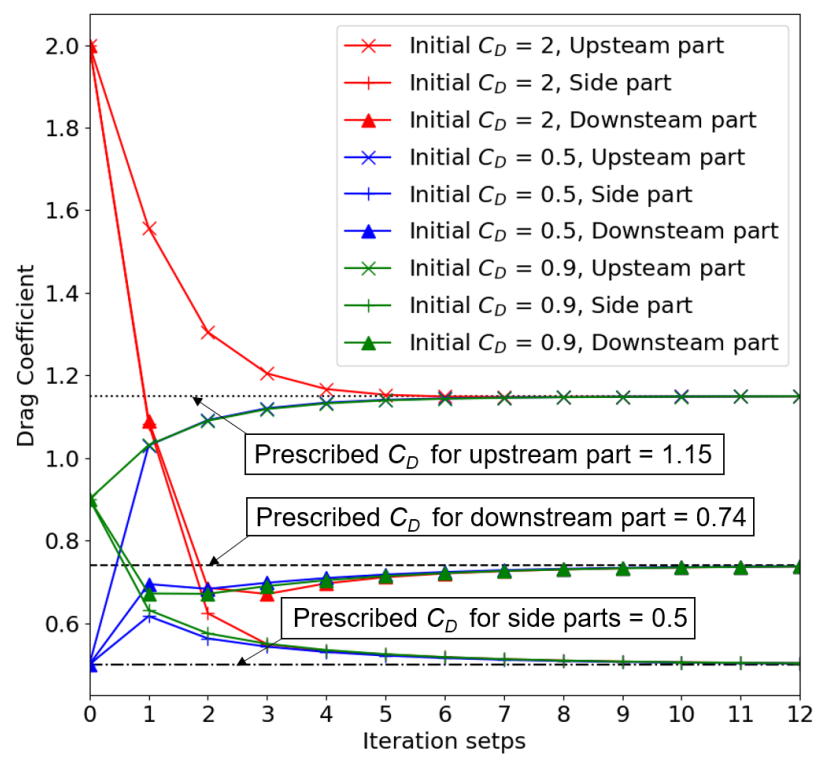

Figure 10: Drag coefficients identified by the PID regulators for the 4-panel configuration.

positions of the fused markers used to regulate their corresponding net panels are illustrated as cross or $\mathrm{x}$ cross, and the rest of the markers are illustrated as square dots. These markers are connected by straight lines showing the general outline. In the moderate flow speed case (Fig. 11), the predicted cage shape from the deterministic model was much less deformed than the measured cage shape in the upstream and middle sections (M4, M7 and M8). In the high flow speed case, the results of all the three numerical models disagreed with the measurement in section near M4. Besides, the predicted shape form 2-panel model did not fit the measured shape well in the middle section (M8), and the deterministic model overpredicted cage deformation in both middle and downstream sections (M8 and M9).

Both the integrated models successfully adjusted the depth of the fused markers to the measured depth, which means the incorporation of measured depths into numerical models was achieved. Besides, both the fused markers and the most unfused markers approached the measured positions, compared with the prediction from the deterministic models. This indicates that the integrated models were able to improve cage deformation prediction holistically rather than only influence the local areas near fused markers.

Fig. 13 compares the prediction accuracy of different models quantitatively. The error distances between measured and predicted positions of M4-M9, as shown in Fig. 11 and 12, were averaged as an indicator of the overall error. The deterministic model had the largest 
averaged error distance, while the 4-panel integrated model had the least in both cases. The performance of the 2-panel and 4-panel models differed in the two cases. In Case 1, the main discrepancy was at the upstream part, and both the integrated models lifted the upstream part to fit the measured deformation. Their prediction accuracy thus was close. By contrast, in Case 2, the 4-panel model was much superior to the 2panel model because the additional fused point at the middle bottom allowed the 4-panel model to fit the less deformed middle section from the measurement.

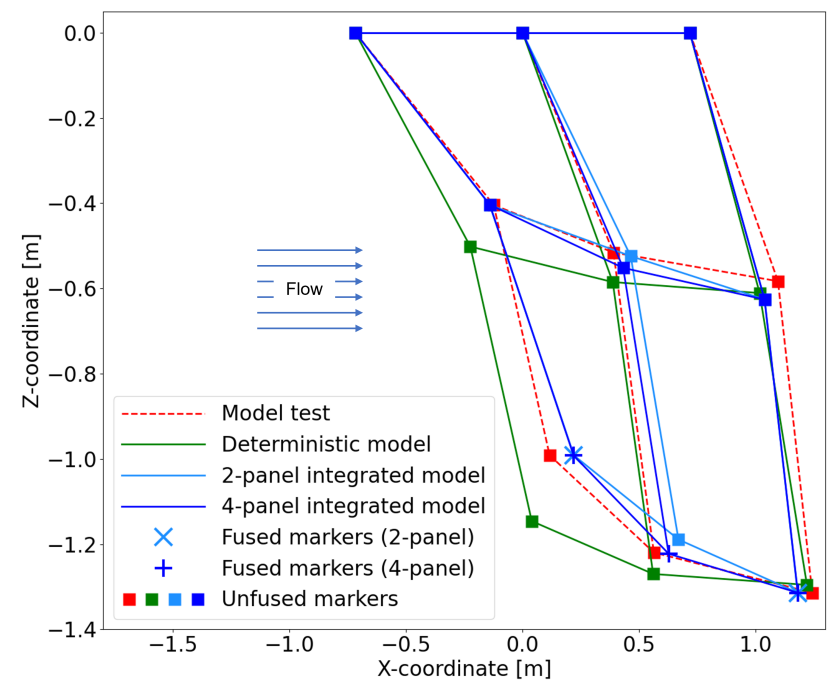

Figure 11: Cage deformation from the model test, deterministic model, and integrated model (Case 1: flow speed $=0.33 \mathrm{~m} / \mathrm{s}$ ).

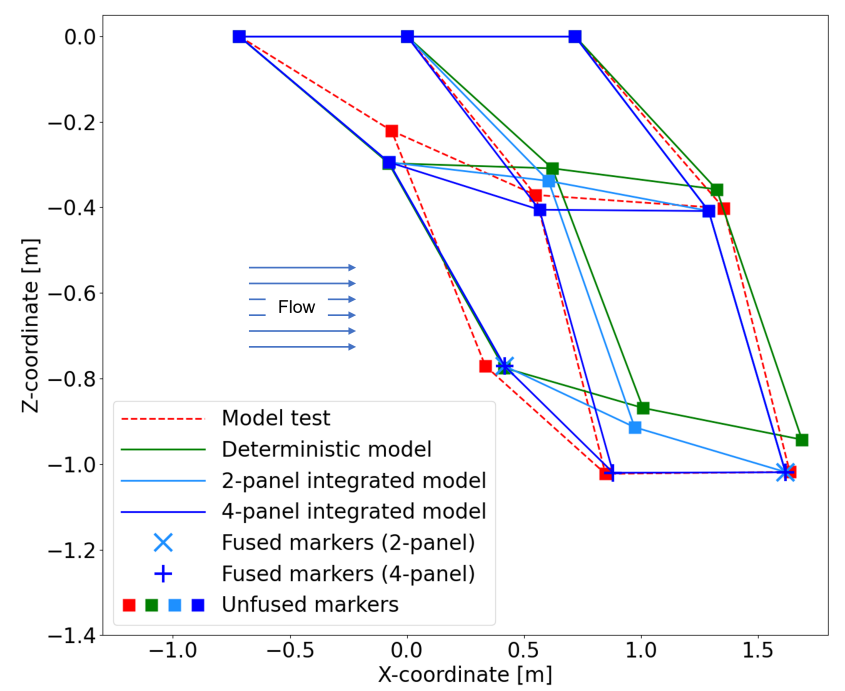

Figure 12: Cage deformation from the model test, deterministic model, and integrated model (Case 2: flow speed $=0.56 \mathrm{~m} / \mathrm{s}$ ).

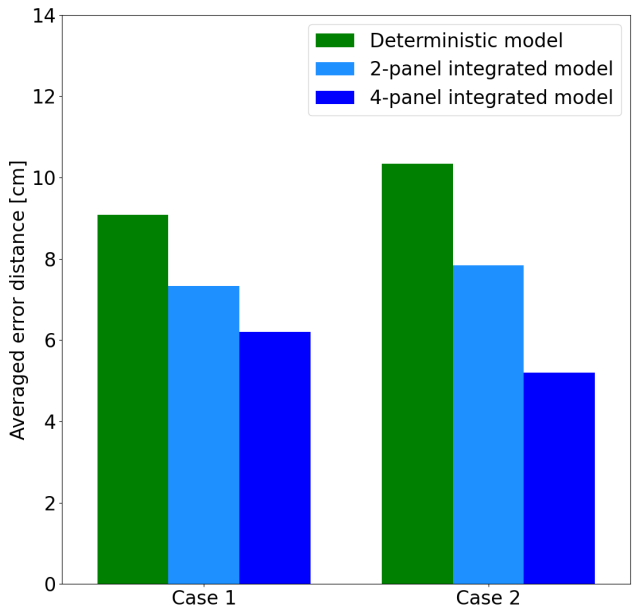

Figure 13: Averaged error distance of the predicted cage deformation from the deterministic models and the integrated models.

\subsection{Fluid loads on the net cage}

Table 4 lists drag coefficient, drag forces, and the flow reduction factors. The estimated drag forces from the integrated models were in a reasonably good agreement with the measured drag forces, with a deviation ratio less than $10 \%$.

Unlike the predefined $C_{D}$ and $C_{D E}$ in the deterministic model, the coefficients of the integrated model were identified by the PID regulators based on measurement. Most of the identified coefficients from the integrated models were close to these in the deterministic model, while the $C_{D}$ of the upstream part of the integrated models in Case 1 was about $65 \%$ larger than the predefined values. Early studies suggest $C_{D}$ for the cage model ranges from 1.20 to 1.26 Tsukrov et al. (2003); Aarsnes et al. (1990) based on different empirical formulas. A model test obtained a $C_{D}$ of 1.5 for a net panel normal to the flow with a solidity of 0.23 Føre et al. (2021), and another experiment on a net panel of 0.21 solidity parallel to the flow gave a $C_{D}$ of 0.56 Zhou et al. (2015). The identified drag coefficients of the front and side parts in Case 2 were within the recommended range from the literature, while the integrated models overestimated these values in Case 1. The overestimation in Case 1 can be explained by the significant discrepancy between the predicted cage shapes from the deterministic model and the cage profile captured in the model test. The measured shape was much more deformed at the upstream part (M7); consequently, the PID regulators resolved a $C_{D}$ larger than that in the deterministic model.

The flow reduction factor $(r)$ can be obtained from $C_{D}$ and $C_{D E}$ following Eq. 2. Previous studies Aarsnes 
et al. (1990); Zhan et al. (2006); Føre et al. (2021); Løland (1993) suggest $r$ ranging from 0.69 to 0.84 . The $r$ resolved by integrated models in Case 2 was within the range, while in Case 1, they were below the range; this should be attributed to the larger $C_{D}$ of the upstream part.

\subsection{Discussion}

This paper investigated the feasibility of incorporating measured depth into a deterministic cage model. Two separate flow speed cases were used in the study to represent the change of current in the real scenario. The proposed integrated models show better performance regarding cage shape prediction than the deterministic model. Both of the integrated models can reach convergence within 15 minutes with a less than $1 \%$ relative error of depth at all the fused positions, which satisfies the requirement of predicting current-induced cage deformation. The prediction accuracy of the proposed integrated models can be influenced by the uncertainties and errors of the deterministic model and the measured data due to its hybrid model nature. On the one hand, the simplification of the deterministic model introduced some errors: the cage was divided into several panels along the circumference, and the $C_{D}$ is approximated to be uniform within a panel. Besides, the mesh grouping method using the truss element cannot completely represent the actual physical properties of the netting Tsukrov et al. (2003). On the other hand, measurement errors of depth can mislead the PID regulators, adversely affecting the prediction of $C_{D}$, drag loads, and cage shapes. When the depth is measured accurately, the integrated models can avoid some uncertainties from deterministic cage models. First of all, the integrated models had high tolerance of the default hydrodynamic parameters. Even if the initial parameters lead to a significant deviation from the measurement, the PID regulators inside the integrated models can adjust these hydrodynamic parameters to narrow down the gap between simulation and measurements. In contrast, selecting proper hydrodynamic parameters for a deterministic model can be tricky. There is no generic formula suitable for all net configurations and flow speeds, and it is also challenging to determine the hydrodynamic parameters among results derived from different formulas. Moreover, the errors from other inputs can also influence the simulation quality in a deterministic model, such as an inaccurate measurement of flow speeds or configurations of the net in the water, while the integrated model can compensate for these errors to some degree.

The present method can be further applied as an essential part of a digital twin of net cages to provide online monitoring and visualizing of net cage deforma- tion. In practice, the depth of full-sized net cages at farm sites can be measured by pressure tags, with typical accuracy of $\pm 0.4 \%$ Klebert et al. (2015). Therefore, when pressure tags are applied in a full-size commercial cage, measurement with good accuracy can be achieved. In addition, the integrated model can better consider the effects of varying environmental and biological factors. The ambient flow and hydrodynamic properties of a cage are dependent on states including incoming current, biofouling, and stocked fish. These states vary in time and spacial domain, which is challenging to measure accurately, let alone to be efficiently considered in the simulations. While in an integrated cage model, its hydrodynamic properties are tuned through the incorporation of measured depth, so that the errors due to the uncertainties of these environmental and biological factors can be reduced. In addition, the hydrodynamic properties contain information regarding the surrounding flow field, such as the flow reduction inside cages. New insights into the flow in and around fish cages can be obtained.

The proposed approach is based on acoustic pressure tags to measure cage deformation, showing advantages in cost and practicalities. A system of ten pressure tags and corresponding acoustic receivers costs about 15,000 NOK per salmon production cycle. When the system is applied to a typical sea cage with 0.17 million salmonids, its cost equals approximately $1 \%$ of the investment of the net cage or $0.1 \%$ of the value of the salmon production; therefore, a small cost-saving or improvement of production can make the application worthy. Moreover, acoustic pressure tags can easily be mounted, for example, sewn into the net directly, due to their simple cylindrical shapes and small sizes (diameter: $2 \mathrm{~cm}$, length: $5 \mathrm{~cm}$ ). Thus, the mounted pressure tags do not disturb farming operations and are not impacted by these operations. The continuous data transmission of acoustic pressure tags also allows for immediate detection if a tag is lost or stops sending data.

\section{Conclusion}

Several sensory techniques have been applied to monitor net cage deformations, and different deterministic models have also been developed to simulate cage deformations under given conditions. However, the measured data cannot give enough information on the overall net cage deformation while the simulation results can be adversely influenced by the imperfection of the deterministic models or unmodeled factors. Thus, both of them have disadvantages when applied to monitor the real shape of fish cages.

The present research investigated the integration of 
Table 4: Summary of the hydrodynamic parameters and fluid forces of model tests Lader and Enerhaug (2005) and numerical simulations.

\begin{tabular}{|c|c|c|c|c|c|c|}
\hline & $\begin{array}{c}C_{D} \\
\text { (Upstream) }\end{array}$ & $\begin{array}{c}C_{D} \\
\text { (Side) } \\
\end{array}$ & $\begin{array}{c}C_{D E} \\
(\text { Downstream) }\end{array}$ & $\begin{array}{c}\text { Drag force } \\
{[\mathrm{N}]}\end{array}$ & $\begin{array}{c}\text { Deviation } \\
{[\%]}\end{array}$ & Flow reduction \\
\hline \multicolumn{7}{|c|}{ Case 1: flow speed $=0.33 \mathrm{~m} / \mathrm{s}$} \\
\hline Model tests & - & - & - & 49.50 & - & - \\
\hline Deterministic model & 1.15 & - & 0.74 & 43.83 & 11.46 & 0.8 \\
\hline Integrated model (2-panel) & 1.86 & - & 0.64 & 52.86 & 6.79 & 0.59 \\
\hline Integrated model (4-panel) & 1.92 & 1.19 & 0.60 & 53.42 & 7.92 & 0.56 \\
\hline \multicolumn{7}{|c|}{ Case 2: flow speed $=0.56 \mathrm{~m} / \mathrm{s}$} \\
\hline Model tests & - & - & - & 87.68 & - & - \\
\hline Deterministic model & 1.15 & - & 0.74 & 81.80 & 6.71 & 0.8 \\
\hline Integrated model (2-panel) & 1.17 & - & 0.59 & 79.03 & 9.86 & 0.71 \\
\hline Integrated model (4-panel) & 1.21 & 0.65 & 0.62 & 78.23 & 9.64 & 0.72 \\
\hline
\end{tabular}

measured depth data with deterministic net cage models using a Morison-type load model for better predictions of cage deformations. Regulators based on PID theory were utilized to tune the hydrodynamic coefficients of a net cage according to deviation between the predicted and measured cage depth. The effectiveness and efficiency of PID regulators to identify net cage's hydrodynamic coefficients were tested in simulation-based tests. Finally, depth data extracted from published model tests Lader and Enerhaug (2005) were incorporated into the cage model and the results shows the present integrated approach can predict cage shapes closer to the measured shapes compared with the knowledge-based model and a finer division of the cage net yields a prediction that fits the measurement better. The predicted fluid loads from the integrated models are also within reasonable accuracy.

This study indicates the feasibility of using regulators of net hydrodynamic parameters to integrate depth (pressure) data with Morison-type cage models, and the proposed method can be applied to achieve online prediction of fish cage deformation at farm sites given updated environmental data and pressure tags data. Due to a lack of field data, the present method was applied in a scaled model test, where the type of sensory technique differs from field measurements. Future work should therefore include a follow-up field experiment on full-sized net cages. Besides, sensor arrangement and calculation efficiency can be further optimized.

\section{Acknowledgements}

This work was jointly financed by the department of Biological Sciences lesund and department of Ocean Operations and Civil Engineering at the Norwegian Uni- versity of Science and Technology. The funding partly came from the R\&D license for salmonids in the sea M-VS-00016.

\section{References}

Aarsnes, J., Rudi, H., and Løland, G. Current forces on cage, net deflection. In Engineering for offshore fish farming, pages 137-152. Thomas Telford Publishing, 1990.

Cheng, H., Li, L., Aarsæther, K. G., and Ong, M. C. Typical hydrodynamic models for aquaculture nets: A comparative study under pure current conditions. Aquacultural Engineering, 2020. 90:102070. doi:10.1016/j.aquaeng.2020.102070.

Cifuentes, C. and Kim, M. Hydrodynamic response of a cage system under waves and currents using a morison-force model. Ocean Engineering, 2017. 141:283-294. doi:10.1016/j.oceaneng.2017.06.055.

Cifuentes, C., Kim, S., Kim, M., and Park, W. Numerical simulation of the coupled dynamic response of a submerged floating tunnel with mooring lines in regular waves. Ocean Systems Engineering, 2015. 5(2):109-123. doi:10.12989/ose.2015.5.2.109.

DeCew, J., Fredriksson, D., Lader, P., Chambers, M., Howell, W., Osienki, M., Celikkol, B., Frank, K., and $\mathrm{H} \varnothing \mathrm{y}$, E. Field measurements of cage deformation using acoustic sensors. Aquacultural engineering, 2013. 57:114-125. doi:10.1016/j.aquaeng.2013.09.006.

Fitridge, I., Dempster, T., Guenther, J., and De Nys, R. The impact and control of biofouling in marine aquaculture: a review. Biofouling, 2012. 28(7):649669. doi:10.1080/08927014.2012.700478. 
Føre, H. M., Endresen, P. C., Norvik, C., and Lader, P. Hydrodynamic loads on net panels with different solidities. Journal of Offshore Mechanics and Arctic Engineering, 2021. 143(5):051901. doi:10.1115/1.4049723.

Gansel, L., Bloecher, N., Floerl, O., and Guenther, J. Quantification of biofouling on nets: a comparison of wet weight measurements and optical (image analysis) methods. Aquaculture international, 2017. 25(2):679-692. doi:10.1007/s10499-016-0062-5.

Gansel, L. C., McClimans, T. A., and Myrhaug, D. Flow around the free bottom of fish cages in a uniform flow with and without fouling. Journal of offshore mechanics and Arctic engineering, 2012. 134(1). doi:10.1115/1.4003695.

Gansel, L. C., Oppedal, F., Birkevold, J., and Tuene, S. A. Drag forces and deformation of aquaculture cagesfull-scale towing tests in the field. Aquacultural Engineering, 2018. 81:46-56. doi:10.1016/j.aquaeng.2018.02.001.

Gansel, L. C., Rackebrandt, S., Oppedal, F., and McClimans, T. A. Flow fields inside stocked fish cages and the near environment. Journal of offshore mechanics and Arctic engineering, 2014. 136(3). doi:10.1115/1.4027746.

He, Z., Faltinsen, O. M., Fredheim, A., and Kristiansen, T. The influence of fish on the mooring loads of a floating net cage. Journal of Fluids and Structures, 2018. 76:384-395. doi:10.1016/j.jfluidstructs.2017.10.016.

Heffernan, D. An introduction to the Python interface to OrcaFlex. Technical report, 2020. URL www . orcina.com/.

Klebert, P., Patursson, Ø., Endresen, P. C., Rundtop, P., Birkevold, J., and Rasmussen, H. W. Threedimensional deformation of a large circular flexible sea cage in high currents: Field experiment and modeling. Ocean Engineering, 2015. 104:511-520. doi:10.1016/j.oceaneng.2015.04.045.

Kristiansen, T. and Faltinsen, O. M. Modelling of current loads on aquaculture net cages. Journal of Fluids and Structures, 2012. 34:218-235. doi:10.1016/j.jfluidstructs.2012.04.001.

Lader, P., Dempster, T., Fredheim, A., and Jensen, $\varnothing$. Current induced net deformations in fullscale sea-cages for atlantic salmon (salmo salar). Aquacultural Engineering, 2008. 38(1):52-65. doi:10.1016/j.aquaculture.2018.06.060.
Lader, P. F. and Enerhaug, B. Experimental investigation of forces and geometry of a net cage in uniform flow. IEEE Journal of Oceanic Engineering, 2005. 30(1):79-84. doi:10.1109/JOE.2004.841390.

Lee, C.-W., Lee, J.-H., Cha, B.-J., Kim, H.Y., and Lee, J.-H. Physical modeling for underwater flexible systems dynamic simulation. Ocean engineering, 2005. 32(3-4):331-347. doi:10.1016/j.oceaneng.2004.08.007.

Løland, G. Current forces on, and water flow through and around, floating fish farms. Aquaculture International, 1993. 1(1):72-89.

Moe, H., Fredheim, A., and Hopperstad, O. Structural analysis of aquaculture net cages in current. Journal of Fluids and Structures, 2010. 26(3):503-516. doi:10.1016/j.jfluidstructs.2010.01.007.

Moe-Føre, H., Christian Endresen, P., Gunnar Aarsæther, K., Jensen, J., Føre, M., Kristiansen, D., Fredheim, A., Lader, P., and Reite, K.-J. Structural analysis of aquaculture nets: comparison and validation of different numerical modeling approaches. Journal of Offshore Mechanics and Arctic Engineering, 2015. 137(4). doi:10.1115/1.4030255.

Moe-Føre, H., Lader, P., Lien, E., and Hopperstad, O. Structural response of high solidity net cage models in uniform flow. Journal of Fluids and Structures, 2016. 65:180-195. doi:10.1016/j.jfluidstructs.2016.05.013.

Priour, D. Calculation of net shapes by the finite element method with triangular elements. Communications in Numerical Methods in Engineering, 1999. 15(10):755-763. doi:10.1002/(SICI)10990887(199910)15:10;755::AID-CNM299;3.0.CO;2-M.

Su, B., Kelasidi, E., Frank, K., Haugen, J., Føre, M., and Pedersen, M. O. An integrated approach for monitoring structural deformation of aquaculture net cages. Ocean Engineering, 2021. 219:108424. doi:10.1016/j.oceaneng.2020.108424.

Tsukrov, I., Eroshkin, O., Fredriksson, D., Swift, M. R., and Celikkol, B. Finite element modeling of net panels using a consistent net element. Ocean Engineering, 2003. 30(2):251-270. doi:10.1016/S00298018(02)00021-5.

Tsukrov, I. I., Ozbay, M., Swift, M. R., Celikkol, B., Fredriksson, D. W., and Baldwin, K. Open ocean aquaculture engineering: numerical modeling. $M a-$ rine Technology Society Journal, 2000. 34(1):29-40. doi:10.4031/MTSJ.34.1.4. 
Turnbull, J., Bell, A., Adams, C., Bron, J., and Huntingford, F. Stocking density and welfare of cage farmed atlantic salmon: application of a multivariate analysis. Aquaculture, 2005. 243(1-4):121-132. doi:10.1016/j.aquaculture.2004.09.022.

Zhan, J., Jia, X., Li, Y. S., Sun, M., Guo, G., and Hu, Y. Analytical and experimental investigation of drag on nets of fish cages. Aquacultural engineering, 2006.
35(1):91-101. doi:10.1016/j.aquaeng.2005.08.013.

Zhou, C., Xu, L., Hu, F., and Qu, X. Hydrodynamic characteristics of knotless nylon netting normal to free stream and effect of inclination. Ocean Engineering, 2015. 110:89-97. doi:10.1016/j.oceaneng.2015.09.043. 